14

\title{
Выявление динамических закономерностей эпилептических приступов у детей методами нелинейной механики
}

\author{
(C) В.Л. Гиляров \\ Физико-технический институт им. А.Ф. Иофрфе РАН, \\ 194021 Санкт-Петербург, Россия \\ e-mail: Vladimir.Hilarov@mail.ioffe.ru
}

Поступило в Редакцию 11 апреля 2019 г.

В окончательной редакции 11 апреля 2019 г.

Принято к публикации 25 сентября 2019 г.

\begin{abstract}
Методами числовых характеристик рекуррентных графиков и мультифрактального анализа исследованы временные ряды главных компонент энцефалограмм детей, страдающих эпилепсией. Показано, что эпилептические приступы сопровождаются увеличением детерминированности процесса электрической активности головного мозга. Это увеличение обусловлено синхронизацией нейронной активности при эпилептических приступах. Отмечено, что такое поведение аналогично поведению нелинейных систем другой физической природы в катастрофических ситуациях.
\end{abstract}

Ключевые слова: нелинейная механика, рекуррентные графики, мультифракталы, эпилепсия.

DOI: 10.21883/JTF.2020.03.48940.164-19

Анализ временных рядов методами нелинейной механики применяется очень широко в различных областях науки: прикладной математике [1-5], физике и механике разрушения материалов [6-10], науках о Земле (геомеханике, геофизике, климатологии) [11-16], биомедицине $[17,18]$, экономике [19-22] и др. При этом используются такие методы как восстановление динамического аттрактора на основании теоремы Такенса, анализ сечений Пуанкаре и расчет показателей Ляпунова [3], модели самоорганизованной критичности [23], рекуррентные графики и их числовые характеристики [24,25], расчет коэффициентов Херста и Гельдера методами нормированного размаха и мультифрактального анализа [26,27].

В настоящей работе используются рекуррентные графики и мультифрактальный метод для анализа временных рядов энцефалограмм (ЭЭГ) детей, страдающих эпилепсией. Данные получены в детской больнице Бостона и опубликованы в базе данных Physionet [18]. Дети наблюдались в течение нескольких суток после отмены противосудорожных препаратов. Сигналы ЭЭГ дискретизировались с частотой $256 \mathrm{~Hz}$ и разрешением 16 bit. Применялась система расположения электродов „10-20“. Кроме того, устанавливались четыре дополнительных электрода $\left(T_{7}, T_{8}, P_{7}, P_{8}\right)$, стандартизованных Американским Электроэнцефалографическим Обществом [28]. Файлы содержали, как правило, часовые записи по 23 каналам (трассам). Иногда добавлялись другие биомедицинские временные ряды (например, электрокардиограммы). Изучались только файлы, содержащие один или несколько эпилептических приступов (база данных содержит времена их начала и окончания). При этом задачами работы не являлись предсказание или раннее обнаружение приступа, а выявление общих закономерностей динамики их развития, если такие существуют.

Сначала исходные энцефалограммы приводились к главным компонентам [29]. Оказалось, что во всех случаях первые десять главных компонент объясняют более $85 \%$ полной дисперсии, поэтому число переменных можно сократить. Пример распределения объясненной дисперсии по главным компонентам приведен на рис. 1.

Далее временные ряды главных компонент разбивались на непересекающиеся интервалы из 1024 сигналов, что при дискретности в 256 bit составляло $4 \mathrm{~s}$. На каждом из таких временных интервалов временной ряд анализировался методом численных характеристик рекуррентных графиков (RQA) и мультифрактальным методом.

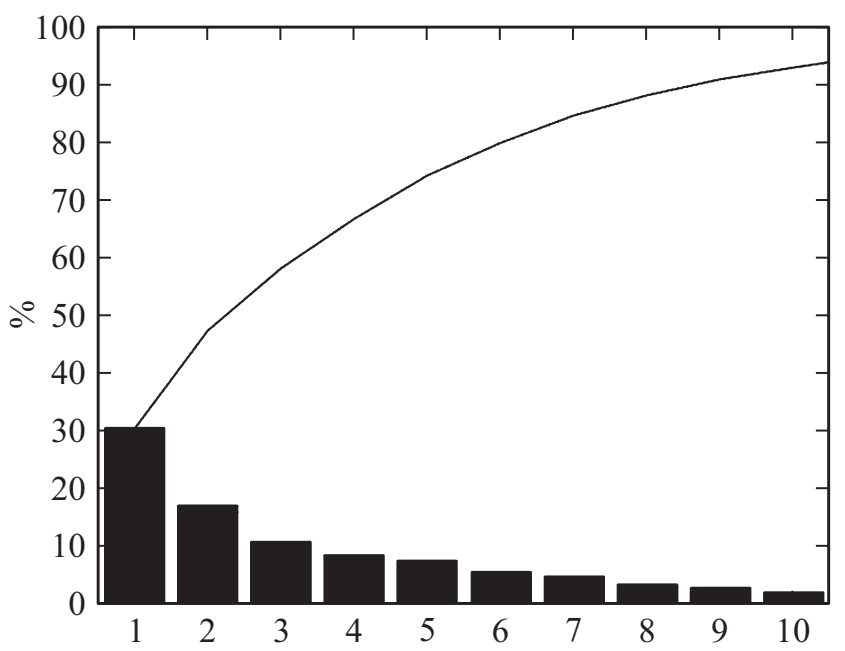

Рис. 1. Пример распределения объясненной полной дисперсии энцефалограмм в \% (ось $y$ ) по главным компонентам. 

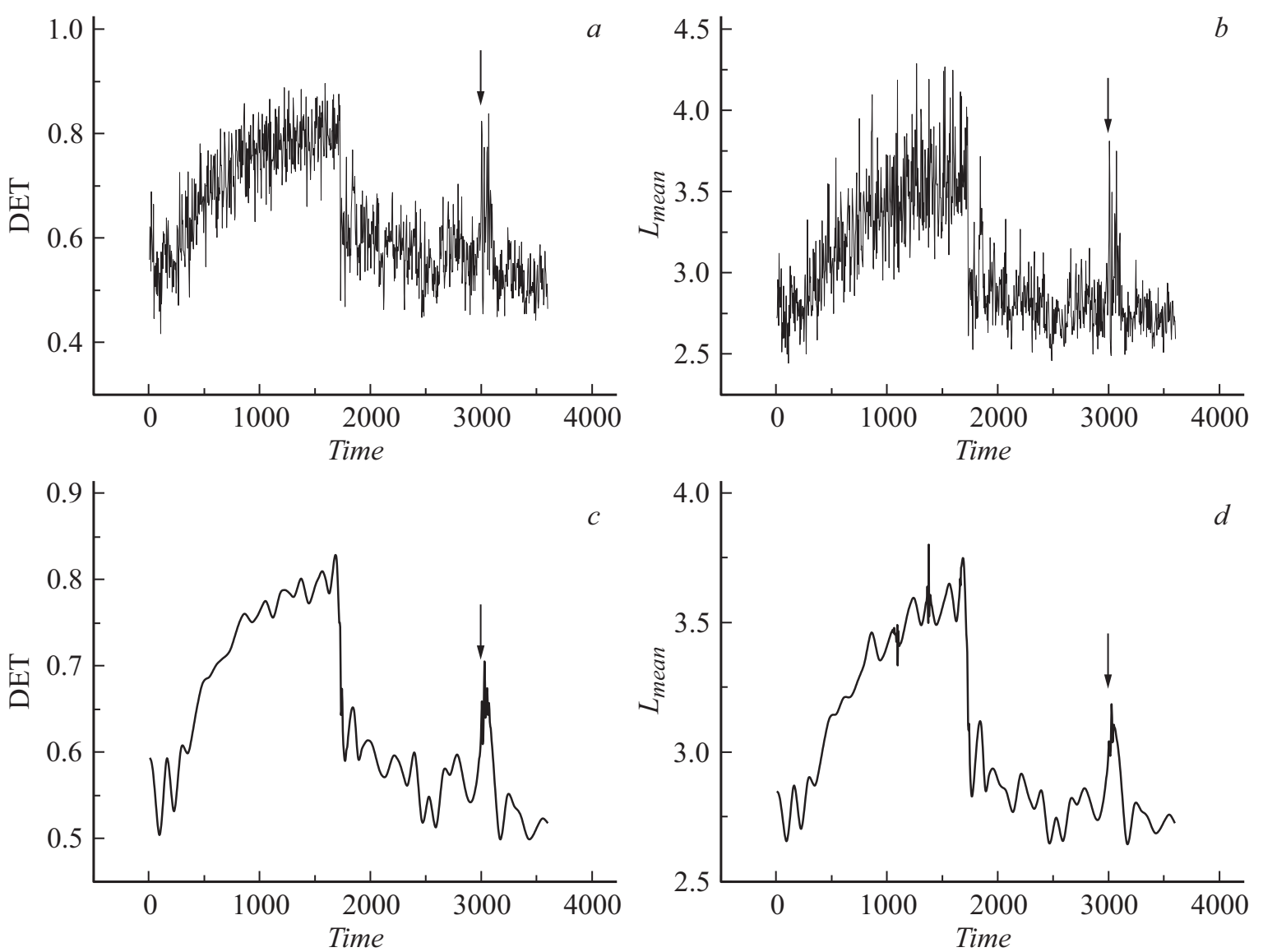

Рис. 2. Зависимости от времени параметров RQA DET $(a, c)$ и $L_{\text {mean }}(b, d)$ для расчетных $(a, b)$ и сглаженных вейвлет фильтром данных $(c, d)$. Стрелками отмечено начало эпилептического приступа (пациент chb01_03).

Для метода RQA строились рекуррентные матрицы вида

$$
R_{i, j}\left(\varepsilon_{i}, m\right)=\theta\left(\varepsilon_{i}-\left\|\mathbf{X}_{i}(m)-\mathbf{X}_{j}(m)\right\|\right)
$$

где $\mathbf{X}_{i}(m)-$ фазовая траектория системы в т-мерном фазовом пространстве, $\theta-$ функция Хевисайда, а \|\|$-$ норма вектора. Рекуррентная матрица, построенная в виде графика, представляет собой двумерное множество точек, отвечающих соотношению (1), состоящее из нулей и единиц, причем близкие (рекуррентные) точки принято отображать черным цветом, а далекие белым. Близость соседей точки $i$ определяется параметром $\varepsilon_{i}$. Выбор больших величин размерности пространства вложения $m$ необходим в случае восстановления аттрактора исходя из теоремы Такенса для скалярных рядов. Так как в настояще работе такая процедура не используется, выбиралось значение $m=1$, поскольку, как показано в обзоре [25], рекуррентные матрицы со значением $m>1$ могут быть выведены из матрицы с $m=1$. Выбор величины порога $\varepsilon_{i}$ существенно влияет на результаты, поэтому обсуждается во многих работах, список которых приведен в [25]. Если выбрать $\varepsilon_{i}$ слишком большим, то точки в основном окажутся близкими друг к другу (рекуррентными), и график будет представлять собой поле черного цвета, а если слишком малым, то рекуррентных точек практически не будет. Согласно рекомендациям [25], величина $\varepsilon_{i}$ выбиралась из условия постоянства плотности рекуррентных точек равной 0.1 (FAN критерий). Для расчетов применялся свободно распространяемый пакет программ CRP Toolbox [25].

Результатом RQA-расчета явились построенные с дискретностью $4 \mathrm{~s}$ временные зависимости числовых параметров рекуррентных графиков. На рис. 2 приведены примеры таких зависимостей для сглаженных и несглаженных величин детерминизма (DET) и средней величины диагональной линии $L_{\text {mean }}$ рекуррентного графика. Величина DET определяется следующим образом.

Сначала вводится в рассмотрение уровень возврата (2):

$$
R R\left(\varepsilon_{i}\right)=\frac{1}{N^{2}} \sum_{i, j=1}^{N} R_{i j}\left(\varepsilon_{i}, m\right) .
$$

Он представляет собой плотность рекуррентных точек на графике. Поскольку в настоящей работе используется алгоритм FAN, сохраняющий постоянной плотность рекуррентных точек, величина $R R \approx 0.1$. Величина детерминизма вводится как отношение числа рекуррентных точек, формирующих диагональные линии, параллель- 

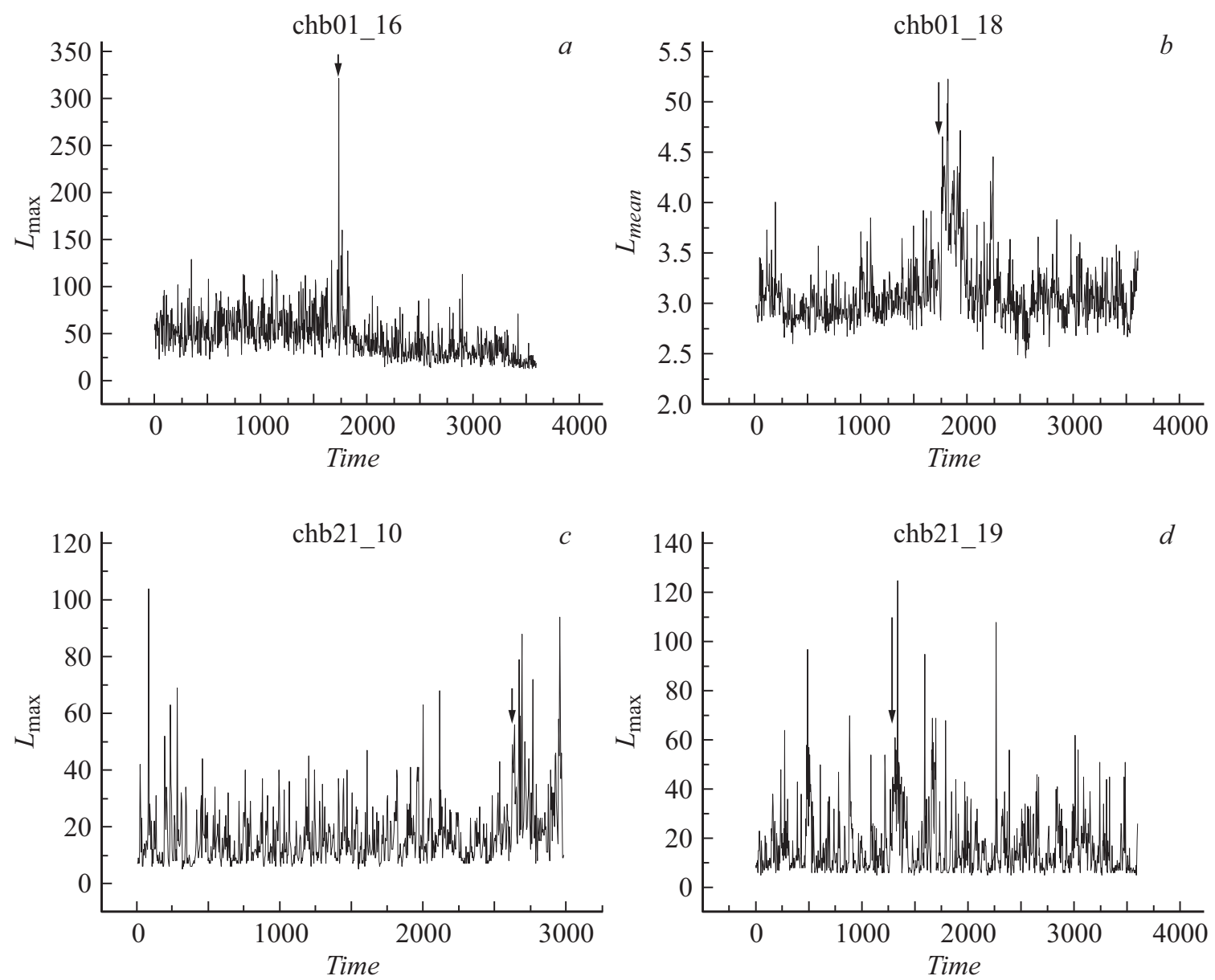

Рис. 3. Зависимости от времени параметров RQA для других эпилептических приступов пациента chb01 (chb01_16, chb01_18); chb21_10 и chb21.19 — того же пациента через 1.5 года.

ные к главной диагонали, к полному числу рекуррентных точек

$$
D E T=\frac{\sum_{i, j=1}^{N} D_{i j}}{\sum_{i, j=1} R_{i j}} .
$$

Хаотические, слабо коррелированные процессы либо вообще не имеют диагональных линий, либо эти линии очень короткие, в то время как детерминированные процессы имеют более длинные диагонали и меньшее число изолированных рекуррентных точек [25].

Сглаживание данных, приведенных на рис. 2 и аналогичных им, проводилось методом удаления высокочастотного шума вейвлет фильтром. Выходной сигнал получался ограничением вейвлет коэффициентов входного сигнала. Использовалось дискретное вейвлет разложение с точностью до 4 порядка. В качестве анализирующего вейвлета выбирался симлет 8 порядка. Применялся мягкий порог с перемасштабированием, зависящим от уровня шума на каждом масштабном уровне [30].

На рис. 2 приведены также времена начала эпилептических приступов. Нетрудно заметить, что эпилептический приступ сопровождается ростом детерминизма
DET и $L_{\text {mean }}$. Графики приведены для третьей главной компоненты (РСA3). Для РСА1 и РСА2 особенности, связанные с эпилептическим приступом, выражены слабо. Это, по-видимому, связано с тем, что в сложной структуре энцефалограммы существуют помимо эпилептических приступов другие схожие особенности (например, веретенообразные шпиндели [31,32]) или артефакты [32]. Следует заметить, что применение вейвлет фильтра низких частот с частотой среза $43 \mathrm{~Hz}$ непосредственно к временным зависимостям главных компонент для устранения наиболее распространенных помех от электромагнитного поля, не внесло каких либо существенных изменений в полученные закономерности, что объясняется тем фактом, что метод главных компонент сам работает как фильтр: информация содержится в основном в первых компонентах, а в более высоких компонентах соотношение сигнал/шум намного меньше.

На рис. 3 представлены временные зависимости параметров RQA для главных компонент энцефалограмм, содержащих другие эпилептические приступы того же пациента (chb01 - девочки 11 лет [33]). Так же на этом рисунке приведены результаты RQA-анализа для той же девочки, снятые полтора года спустя. Во всех 

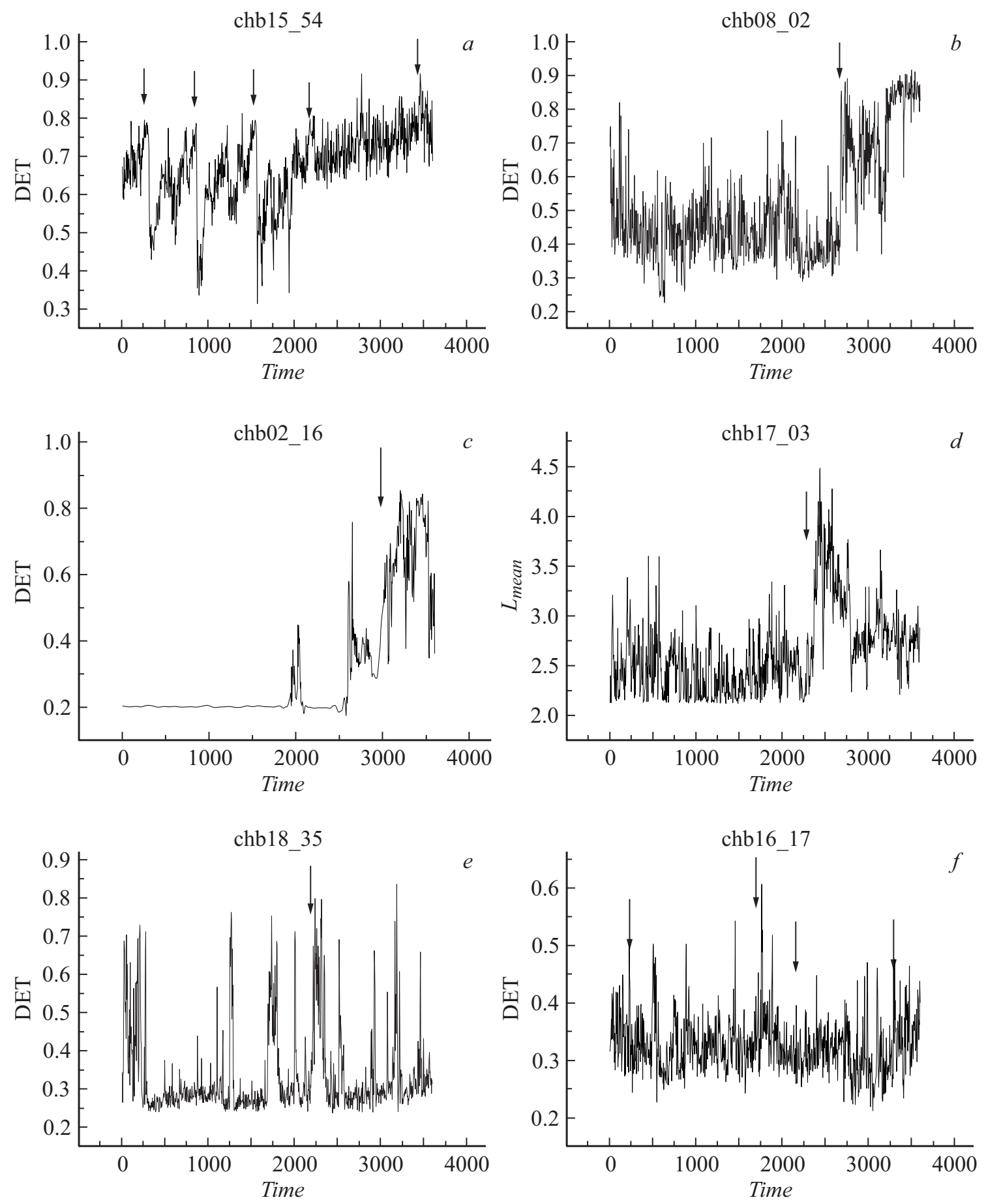

Pис. 4. Зависимости от времени параметров RQA для эпилептических приступов других пациентов.

случаях наблюдается увеличение детерминированности процесса, сопровождающее эпилептические приступы.

На рис. 4 представлены параметры RQA для других пациентов. Вновь можно отметить, что эпилептические приступы сопровождаются увеличением детерминированности процесса. Следует отметить, что в редких случаях наблюдалась немного иная закономерность (рис. 5). Если уровень детерминизма первых главных компонент энцефалограмм пациента был достаточно высок
$(D E T \approx 0.9)$, то эпилептическому приступу предшествовало уменьшение параметров, отражающих диагональные структуры рекуррентных графиков. Однако они возвращались к своим высоким значениям или прямо во время приступа или немедленно после него.

На рис. 6 приведены зависимости от времени положения максимума мультифрактального спектра сингулярностей (глобального коэффициента Херста) главных компонент ряда энцефалограмм, содержащих сер- 

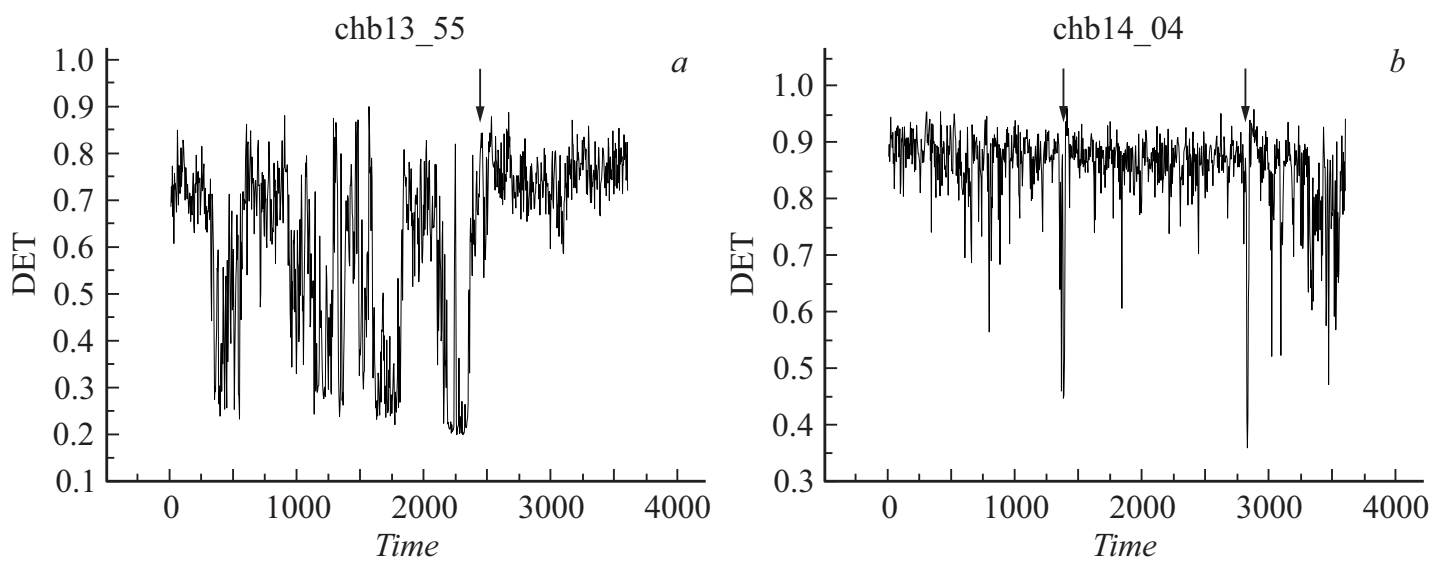

Pис. 5. Зависимость от времени параметров RQA для пациентов с высоким уровнем детерминированности энцефалограмм.
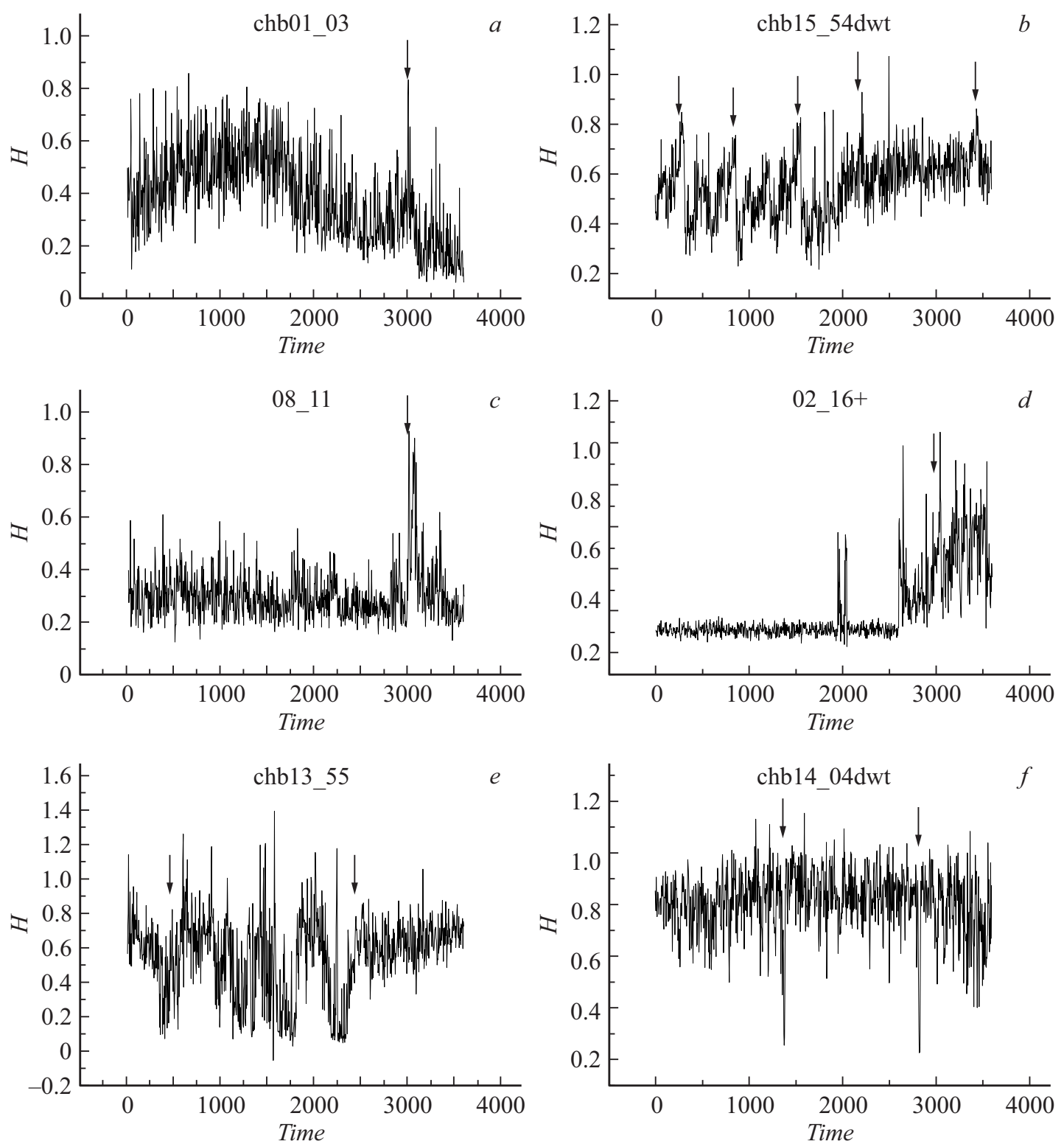

Рис. 6. Зависимость от времени глобального коэффициента Херста главных компонент энцефалограмм (РСА3 и РСА1 (e)), содержащих эпилептические приступы. 
дечные приступы. Для расчета спектра сингулярностей использовался метод вейвлет лидеров [34,35]. Вейвлет лидер $L(j, k)$ представляет собой наибольший коэффициент дискретного вейвлет преобразования $d\left(j^{\prime}, k^{\prime}\right)$, вычисленный в узком временном интервале $(k-1) \cdot 2^{j} \leq k^{\prime} \cdot 2^{j^{\prime}} \leq(k+1) \cdot 2^{j} \quad$ на всех масштабах, меньших заданного $w^{j^{\prime}} \leq 2^{j}$. Вейвлет лидеры отражают локальные коэффициенты Гельдера, которые можно рассчитать стандартным для мультифрактального анализа способом: построить обобщенную функцию распределения вида $Z(j, q) \propto \sum_{k} L(j, k)^{q} \propto 2^{j \cdot \tau(q)}$, найти из нее скейлинговые экспоненты $\tau(q)$, из которых рассчитать спектр сингулярностей $D(h)$ при помощи преобразования Лежандра. Таким образом, метод вейвлет лидеров можно считать дальнейшим развитием метода максимума модуля вейвлет преобразования, разработанного в [36].

Графики, приведенные на рис. 6, $a-d$ отражают общую закономерность - рост коэффициента Херста, сопровождающий эпилептические приступы. Графики на рис. $6, e, f$ соответствуют кривым на рис. 5 , когда эпилептическому приступу предшествует хаотизация процесса. Но самим приступам вновь сопутствует увеличение коэффициента Херста.

Подобные закономерности наблюдаются при других видах аномального поведения сложных нелинейных систем и катастрофических явлениях. Мультифрактальные спектры сердечного ритма у здоровых людей более широкие, а коэффициенты Херста меньше, чем у людей, страдающих сердечными заболеваниями (хронической сердечной недостаточностью или уменьшением систолической функции левого желудочка) [37,38]. Мультифрактальные спектры сингулярностей поверхностных профилей металлов и аморфных сплавов, измеренные методами зондовой микроскопии, трансформируются в процессе механического нагружения и сужаются перед разрушением материала $[39,40]$. Известно, что сильным землетрясениям и горным ударам предшествуют уменьшение фрактальной размерности (увеличение коэффициента Херста) [41], а также уменьшение ширины спектра сингулярностей сейсмических временных рядов [42] и увеличение детерминированности процесса по данным RQA [43]. Такие же эффекты были отмечены во временных рядах акустической эмиссии при формировании магистральной трещины в лабораторных образцах [44]. Для процесса разрушения материалов изученные закономерности обусловлены коллективизацией дефектов структуры на разных масштабных уровнях, приводящей к фрактальной самоорганизации процесса. Известно [33], что при эпилептических приступах происходит синхронизация нейронной активности. Таким образом, и в этом случае происходит коллективизация структурных элементов в процессе электрической активности головного мозга.

Полученные результаты свидетельствуют о том, что эпилептические приступы сопровождаются увеличени- ем детерминированности процесса, проявляющейся в увеличении параметров, связанных с диагональными линиями рекуррентных графиков, а также увеличением глобальных коэффициентов Херста (увеличением персисентности). Таким образом, характер процесса изменяется от более сложного к более простому. Следует отметить, что изменения детерминированности процесса, как следует из рис. 2-6, не обязательно связаны с эпилептическими приступами. Т.е. эти изменения представляют собой только необходимые, но не достаточные условия проявления эпилепсии.

\section{Конфликт интересов}

Автор заявляет, что у него конфликта интересов.

\section{Список литературы}

[1] Моисеев Н.Н. Ассимптотические методы нелинейной механики. М.: Наука, 1981. 400 с.

[2] Katok A., Hasselblatt B. Introduction to the Modern Theory of Dynamical Systems. Cambridge: Cambridge University Press, 1995. 824 p.

[3] Малинецкий Г.Г., Потапов А.Б. Современные проблемы нелинейной динамики. М.: Эдиториал УРСС, 2000. 335 c.

[4] Olson C.C., Nichols J.M., Virgin L.N. // Nonlinear Dynamics. 2012. Vol. 70. N 1. P. 381-391.

DOI: $10.1007 / \mathrm{s} 11071-012-0461-8$

[5] Hegger R., Kantz H., Schreiber T. // Chaos. 1999. Vol. 9. N 2. P. 413-434. DOI: $10.1063 / 1.166424$

[6] Nichols J.M., Trickey S.T., Seaver M. // Mech. Syst. Signal Process. 2006. Vol. 20. N 2. P. 421-437. DOI: $10.1016 /$ j.ymssp.2004.08.007

[7] Nichols J.M., Seavera M., Trickeya S.T. // J. Sound and Vibration. 2006. Vol. 297. N 1-2. P. 1-16. DOI: $10.1016 /$ j.jsv.2006.01.025

[8] Гиляров В.Л. // ФТТ. 2015. Т. 57. Вып. 11. С. 2204-2211. [Hilarov V.L. // Solid State Phys. 2015. Vol. 57. N 11. P. 2271-2278.] DOI: 10.1134/S1063783415110116

[9] Смирнов В.Б., Пономарев А.В., Завьялов А.Д. // Физика Земли. 1995. Вып. 1. Р. 38-58. [Smirnov V.B., Ponomarev A.V., Zavyalov A.D. // Fizika Zemli. 1995. N 1. P. 38-58.]

[10] Kacimi S., Laurens S. // J. Appl. Phys. 2009. Vol. 106. N 2. P. 024909. DOI: $10.1063 / 1.3169601$

[11] Золотова Н.В., Понявин Д.И. // Уч. зап. СПбГУ 2005. № 438 (Вопр. геофизики). Вып. 38. С. 203-231.

[12] Захаров В.С. // Нелинейный мир. 2010. Т. 8. Вып. 4. C. 234-241.

[13] Lyubushin A.A. // J. Seismology. 2015. Vol. 19. N 2. P. 329-340. DOI: 10.1007/s10950-014-9468-6

[14] Смирнов В.Б., Пономарев A.B., Jiadong Q., Черепанцев А.С. // Физика Земли. 2005. № 6. С. 6-28.

[15] Lei X.L., Nishizawa O., Kusunose K. // Geophys. J. Int. 1993. Vol. 115. N 1. P. 79-84. DOI: $10.1111 / j .1365-246 X .1993 . t b 05589 . x$

[16] Макаров П.В., Еремин М.О. // Физ. Мезомеханика. 2016. Т. 19. Вып. 16. С. 62-76. 
[17] Меклер А.А. Вестн. новых медицинских технологий. 2007. T. 14. Вып. 1. С. 73-76.

[18] Goldberger A.L., Amaral L.A.N., Glass L., Hausdorff J.M., Ivanov P.Ch., Mark R.G., Mietus J.E., Moody G.B., Peng C.-K., Stanley H.E. // Circulation. 2000. Vol. 101. N 23. P. E215-E220. DOI: 10.1161/01.CIR.101.23.e215

[19] Fabretti A., Ausloos M. // Int. J. Mod. Phys. C. 2005. Vol. 16. N 5. P. 671-703. DOI: $10.1142 / \mathrm{S} 0129183105007492$

[20] Modelling and Forecasting Financial Data / Ed. Soofi A.S., Liangyue C. Springer, 2002. 481 p.

[21] Nonlinear Economic Dynamics and Financial Modelling. / Ed. Dieci R., He X.-Z., Hommes C. Springer, 2014. 389 p.

[22] Faggini M. // Chaos. 2014. Vol. 24. N 4. P. 042101. DOI: $10.1063 / 1.4903797$

[23] Turcotte D.L. // Rep. Prog. Phys. 1999. Vol. 62. N 10. P. $1377-1429$. DOI: $10.1088 / 0034-4885 / 62 / 10 / 201$

[24] Eckmann J.-P., Kampost S.O., Ruelle D. // Europhys. Lett. 1987. Vol. 4. N 9. P. 973-977.

[25] Marwan N., Romano M.C., Thiel M., Kurths J. // Phys. Rep. 2007. Vol. 438. N 5-6. P. 237-329.

DOI: 10.1016/j.physrep.2006.11.001

[26] Федер Е. Фракталы. М.: Мир, 1991. 254 с.

[27] Павлов А.Н., Анищенко В.С. // УФН. 2007. Т. 177. Вып. 8. C. $859-876$.

[28] Sharbrough F., Chatrian G.-E., Lesser R.P., Lüders H., Nuwer M., Picton T.W. // J. Clin. Neurophysiol. 1991. Vol. 8. N 2. P. 200-202.

[29] Эсбенсен К. Анализ многомерных данных. Черноголовка: Изд-во: ИПХФ РАН, 2005. 160 с.

[30] Дьяконов В.П. Вейвлеты. От теории к практике. М.: СОЛОН-Пресс, 2010. 400 c.

[31] Kharbouch A., Shoeb A., Guttag J. // Epilepsy and Behavior. 2011. Vol. 22. Suppl. 1. P. S29-S35. DOI: 10.1016/j.yebeh.2011.08.031

[32] Зенков Л.Р. Клиническая электроэнцефалография (с элементами эпилептологии). Руководство для врачей. М.: МЕДпрессинформ, 2017. 360 с.

[33] Shoeb A. // PhD Thesis, Massachusetts Institute of Technology, September 2009, 162 p.

[34] Wendt H., Abry P., Jaffard S. // IEEE Signal Process. 2007. Vol. 24. N 4. P. 38-48. DOI: 10.1109/MSP.2007.4286563

[35] Wendt H., Roux S.G., Jaffard S., Abry P. // Signal Process. 2009. Vol. 89. N 6. P. $1100-1114$. DOI: $10.1016 /$ j.sigpro.2008.12.015

[36] Muzy J.F., Bacry E., Arneodo A. // Phys. Rev. 1993. Vol. E47. N 2. P. $875-884$. DOI: $10.1103 /$ PhysRevE.47.875

[37] Ivanov P.Ch., Amaral N.L.A., Goldberger A.L., Havlin S., Rosenblum M.G., Struzikk Z.R., Stanley H.E. // Nature. 1999. Vol. 399. N 6735. P. 461-465. DOI: 10.1038/20924

[38] Makowiec D., Galaska R., Dudkowska A., Rynkiewicz A., Zwierz M. // Physica. 2006. Vol. A369. N 2. P. 632-644. DOI: $10.1016 /$ j.physa.2006.02.038

[39] Гиляров В.Л., Корсуков В.Е., Бутенко П.Н., Светлов В.Н. // ФТТ. 2004. Вып. 10. С. 1806-1810.

[40] Корсуков В.Е., Князев С.А., Бутенко П.Н., Гиляров В.Л., Корсукова М.М., Няпшаев И.А., Обидов Б.А. // ФТТ. 2017. т. 59. Вып. 2. C. 308-311. DOI: http://dx.doi.org/10.21883/FTT.2017.02.44053.269

[41] Advances in Geophysics Ed. R. Dmowska, B. Saltzman. Vol. 44. Elsevier, 2000. 188 p.

DOI: $10.1016 / \mathrm{s} 0065-2687(00) 80007-2$
[42] Касимова В.А., Копылова Г.Н., Любушин А.А. // Физика Земли. 2018. Вып. 2. С. 81-95. DOI: $10.7868 / \mathrm{S} 0002333718020072$

[43] Гиляров В.Л., Дамаскинская Е.Е., Рассказов И.Ю. // Проблемы недропользования. 2016. Вып. 2. С. 53-57. DOI: $10.18454 / 2313-1586.2016 .02 .05$

[44] Дамаскинская Е.Е., Гиляров В.Л., Пантелеев И.А., Гафурова Д.Р., Фролов Д.И. // ФТТ. 2018. Т. 60. Вып. 9. C. $1775-1780$. DOI: $10.21883 /$ FTT.2018.09.46396.078 